Age and maturity, changes in the balance of water and electrolytes in the brain, and various drugs were factors found to modify the threshold convulsive temperature in young animals. An antihistamine, diphenhydramine and the anticonvulsant, phenytoin lowered the threshold convulsive temperature and exacerbated fever-induced seizures, whereas phenobarbital and phetharbital elevated the threshold and prevented seizures. (Millichap JG. Febrile Convulsions, Macmillan, New York, 1968). See Progress in Pediatric Neurology II, 1994, pp16-32, and I, 1991, pp14-24, (edited by Millichap, PNB

Publishers) for a compendium of more current articles on febrile seizures.

\title{
SUPPLEMENTARY SENSORIMOTOR SEIZURES
}

The diagnosis, clinical features, video EEG and MRI findings, medical and surgical treatment, pathology, and prognosis in eleven children and adolescents with supplementary sensorimotor area seizures (SSMA) are reported from the Departments of Neurology, Neurosurgery, and Radiology, Cleveland Clinic Foundation, Cleveland, Ohio. Mean age at onset was 5.8 years, and the diagnosis was made by vertex sharp waves on prolonged video EEG ( 3 to 7 days) at a mean age of 12 years. Neurologic exam was normal, except for 2 patients with a focal decrease in hand coordination, and routine EEGs were frequently normal. Seizures were usually bilateral and tonic, affecting proximal limb muscles, frequent, occurring daily, refractory to medication, without loss of cosciousness, and mainly during sleep. MRI revealed a lowgrade tumor or focal cortical dysplasia in 5 patients. Six had cortical resection after confirmation of SSMA by subdural EEG, and 5 were benefited. (Bass $\mathrm{N}$, Wyllie E et al. Supplementary sensorimotor area seizures in children and adolescents. I Pediatr April 1995;126:537-544). (Reprints: Elaine Wyllie MD, Head, Pediatric Epilepsy Program, Cleveland Clinic Foundation, Desk S51, 9500 Euclid Ave, Cleveland, OH 44195).

COMMENT. SSMA seizures differ from generalized TC seizures in preservation of consciousness, and from perirolandic benign focal epilepsy of childhood in bilaterality and proximal gross flailing movements. Preserved consciousness and gross bilateral, proximal limb movements are the principal distinguishing features of SSMA. Brevity and nocturnal predominance are other characteristic features.

Prolonged video EEG and MRI are important in diagnosis, and surgery should be considered in refractory patients. The neuropsychological and behavioral abnormalities often found in adolescents with frontal lobe seizures or damage (see Progress in Pediatric Neurology I, 1991, p71, and Vol II, 1994, p180-3) were not evident in these patients with lesions in the supplementary SM area. Auras and sensory manifestations may be more difficult to elicit in children than in adults. Auras in 5 of the above patients included crawling, tingling or heavy sensations of the limbs and one complained of epigastric discomfort. Similar sensations were described in case reports of Penfield W, and Jasper H. Epilepsy and the Functional Anatomy of the Human Brain. Little, Brown, Boston, 1954, p398.

\section{REFLEX MYOCLONIC EPILEPSY OF INFANCY}

Six neurologically normal infants, aged 6-21 months, with attacks resembling benign myoclonic epilepsy of infancy but occurring as reflex 
responses to auditory and tactile stimuli are reported from Bambino Gesu Children's Hospital, Rome, Italy. An excessive startle response caused symmetric jumping of limbs, arms more than legs. Spontaneous attacks developed in 4, particularly in sleep. A family history of epilepsy or febrile convulsions was elicited in 5. Remissions occurred in 4-14 months, spontaneously or in response to valproate. EEGs showed short generalized spike- or polyspike-and-wave discharges. (Ricci S et al. Reflex myoclonic epilepsy in infancy: A new age-dependent idiopathic epileptic syndrome related to startle reaction. Epilepsia April 1995;36:342-348). (Reprints: Dr S Ricci, Section of Neurophysiology, Bambino Gesu Children's Hospital, IRCCS, Piazza S Onofrio 4, 00165 Rome, Italy).

COMMENT. In addition to the reflex nature of these attacks, the abnormal EEG appears to distinguish this syndrome from benign myoclonic epilepsy in infancy. Therapy with anticonvulsants seemed justified when attacks occurred spontaneously and frequently, from 5 to 20 times daily.

\section{READING EPILEPSY}

The electroclinical manifestations and natural history of reading epilepsy (RE) in 20 patients diagnosed between 1949 and 1989 are reported from the Mayo Clinic, Rochester, Minnesota. Age at onset ranged from 10 to 46 years (median 17 years). Juvenile myoclonic epilepsy occurred in 4 , and a positive family history for epilepsy in 4, with RE in 1 . Seizures were myoclonic, involving orofacial and jaw muscles, and the upper limbs also in 5 . Generalized tonic-clonic seizures occurred at least once in 16. The EEG showed generalized spike or spike-and wave discharges in 15 cases and left hemisphere discharges in 5. RE was persistent into late adult life but not progressive; it responded to valproic acid. Higher cognitive processes acting as trigger mechanisms other than reading included calculation in 6 , speaking under stress in 5, writing in 2, and playing chess in 1. (Radhakrishnan K, Silbert PL, Klass DW. Reading epilepsy. An appraisal of 20 patients diagnosed at the Mayo Clinic, Rochester, Minnesota, between 1949 and 1989, and delineation of the epileptic syndrome. Brain Feb 1995;118:75-89). (Respond: Donald W Klass MD, Section of Electroencephalography, Mayo Clinic, 200 First Street SW, Rochester, MN 55905).

COMMENT. The authors dedicate their article to Dr Reginald G Bickford on his 81st birthday and we add our congratulations! Bickford (1954) and Bickford, Klass et al (1956) first described the syndrome of reading epilepsy and stressed the importance of precipitating factors in the mechanism of seizures and EEG epileptiform discharges in general. Christie S (1988) found a combination of factors involved in the precipitation of reading epilepsy: saccadic eye movements, articulation, and difficulty of linguistic content. Bickford had alluded to the degree of difficulty of reading matter in his original article. (See Progress in Pediatric Neurology I, 1991, p45).

\section{PREVENTION OF EPILEPSY AND COMPLICATIONS}

Prevention of epilepsy and its consequencies is discussed in a special article from the Department of Neurology, School of Medicine, University of Virginia, Charlottesville. Prevention should apply at various levels: 1 . epileptogenesis may be prevented by a) avoidance of premature birth, in 